\title{
Abnormal pulmonary function in COVID-19 patients at time of hospital discharge
}

\author{
To the Editor:
}

On 11 March 2020, the World Health Organization (WHO) declared coronavirus disease 2019 (COVID-19) as a pandemic. As of 22 April, more than 2.4 million cases have been confirmed worldwide [1]. In light of the widely documented lung injuries related to COVID-19 [2, 3], concerns have been raised regarding the assessment of lung injury for discharged patients. A recent report portrayed that discharged patients with COVID-19 pneumonia still have residual abnormalities in chest computed tomography (CT) scans, with ground-glass opacity as the most common pattern [4]. Persistent impairment of pulmonary function and exercise capacity have been known to last for months or even years [5-8] in the recovered survivors from other coronavirus pneumonia (severe acute respiratory syndrome (SARS) and Middle East respiratory syndrome (MERS)). However, until now, there is no report in regard to pulmonary function in discharged COVID-19 survivors. This article aims to describe the characteristics of pulmonary function in these subjects.

We recruited laboratory-confirmed noncritical COVID-19 cases, from 5 February to 17 March 2020, from admitted patients. According to the WHO interim guidance [9] and the guidance from China [10], disease severity was categorised as mild illness (mild symptoms without radiographic appearance of pneumonia), pneumonia (having symptoms and the radiographic evidence of pneumonia, with no requirement for supplemental oxygen), severe pneumonia (having pneumonia, including one of the following: respiratory rate $>30$ breaths. $\mathrm{min}^{-1}$; severe respiratory distress; or oxygen saturation measured by pulse oximetry $\left(S_{\mathrm{pO}_{2}}\right)$ $\leqslant 93 \%$ on room air at rest), and critical cases (e.g. respiratory failure requiring mechanical ventilation, septic shock, other organ failure occurrence or admission into the intensive care unit). Critical cases were excluded from our study. Spirometry and pulmonary diffusion capacity tests (Quark PFT; Cosmed, Rome, Italy) were performed following the American Thoracic Society/European Respiratory Society guidelines on the day of or 1 day before discharge. To minimise cross-infections, diffusing capacity of the lung for carbon monoxide $\left(D_{\mathrm{LCO}}\right)$ was measured by the single-breath method. Written informed consent was obtained from all patients, and the study was approved by the ethics committee of The Guangzhou Eighth People's Hospital.

110 discharged cases were recruited, which included 24 cases of mild illness, 67 cases of pneumonia and 19 cases of severe pneumonia (table 1). The mean age of these cases was 49.1 years and 55 of them were females. 44 patients (40\%) had at least one underlying comorbidity, of which $23.6 \%$ had hypertension and $8.2 \%$ had diabetes. Only three patients $(2.7 \%)$ were reported as having chronic respiratory diseases (one patient with asthma, one with chronic bronchitis and one with bronchiectasis). No significant differences were found among the three groups of cases in relation to sex, smoking status, underlying disease and body mass index. The mean \pm SD duration from onset of disease to pulmonary function test was $20 \pm 6$ days in cases with mild illness, $29 \pm 8$ days in cases with pneumonia and $34 \pm 7$ days in cases that presented severe pneumonia. On the day of discharge, the $S_{\mathrm{pO}_{2}}$ on room air at rest was normal in all subjects and no significant difference was found among the different groups (all $\mathrm{p}>0.05$ ).

Spirometry was uneventfully completed in all patients, except for two failed diffusion capacity tests. Anomalies were noted in $D_{\text {LCO }} \%$ predicted in 51 cases $(47.2 \%)$, total lung capacity (TLC) \% pred in 27 $(25.0 \%)$, forced expiratory volume in $1 \mathrm{~s}\left(\mathrm{FEV}_{1}\right) \%$ pred in $15(13.6 \%)$, forced vital capacity (FVC) \% pred in 10 (9.1\%), $\mathrm{FEV}_{1} / \mathrm{FVC}$ in five (4.5\%), and small airway function in eight (7.3\%). Table 1 shows a significant difference in impaired diffusing capacity among the different groups of severity, which accounted for $30.4 \%$ in mild illness, $42.4 \%$ in pneumonia and $84.2 \%$ in severe pneumonia, respectively $(\mathrm{p}<0.05)$.

@ERSpublications

In discharged survivors with COVID-19, impairment of diffusion capacity is the most common abnormality of lung function, followed by restrictive ventilatory defects, which are both associated with the severity of the disease https://bit.ly/2yUaBaT

Cite this article as: Mo X, Jian W, Su Z, et al. Abnormal pulmonary function in COVID-19 patients at time of hospital discharge. Eur Respir J 2020; 55: 2001217 [https://doi.org/10.1183/13993003.01217-2020]. 
TABLE 1 Demographics and pulmonary function characteristics of discharged patients with COVID-19

\begin{tabular}{|c|c|c|c|c|c|}
\hline & Total & Mild illness & Pneumonia & $\begin{array}{c}\text { Severe } \\
\text { pneumonia }\end{array}$ & p-value \\
\hline Patients & 110 & 24 & 67 & 19 & \\
\hline Age years & $49.1 \pm 14.0$ & $46.8 \pm 15.6$ & $47.9 \pm 13.7$ & $56.5 \pm 11.0^{*} \#$ & 0.04 \\
\hline Female & $55(50.0)$ & 13 (54.2) & $36(53.7)$ & $6(31.6)$ & 0.21 \\
\hline Smoker & 13 (11.8) & $4(16.7)$ & $7(10.4)$ & $2(10.5)$ & 0.707 \\
\hline BMI $\mathrm{kg} \cdot \mathrm{m}^{-2}$ & $23.5 \pm 3.0$ & $23.1 \pm 2.8$ & $23.6 \pm 3.2$ & $23.5 \pm 2.7$ & 0.794 \\
\hline $\begin{array}{l}\text { Duration from onset to } \\
\text { discharge days }\end{array}$ & $27 \pm 9$ & $20 \pm 6$ & $29 \pm 8^{* *}$ & $34 \pm 7^{* *, \#}$ & $<0.001$ \\
\hline Underlying disease & $44(40.0)$ & $10(41.7)$ & 25 (37.3) & $9(47.4)$ & 0.719 \\
\hline Lung disease & $3(2.7)$ & $0(0)$ & $3(4.5)$ & $0(0)$ & 1 \\
\hline Heart disease & $3(2.7)$ & $1(4.2)$ & $2(3.0)$ & $0(0)$ & 1 \\
\hline Hypertension & $26(23.6)$ & $6(25.0)$ & $15(22.4)$ & $5(26.3)$ & 0.924 \\
\hline Cerebrovascular disease & $3(2.7)$ & $0(0)$ & $2(3.0)$ & $1(5.3)$ & 0.532 \\
\hline Diabetes & $9(8.2)$ & $1(4.2)$ & $6(9.0)$ & 2 (10.5) & 0.702 \\
\hline Liver disease & $6(5.5)$ & 2 (8.3) & $3(4.5)$ & $1(5.3)$ & 0.837 \\
\hline Kidney disease & $2(1.8)$ & $1(4.2)$ & $1(1.5)$ & $0(0)$ & 0.631 \\
\hline Solid tumour & $1(0.9)$ & $0(0)$ & $0(0)$ & $1(5.3)$ & 0.173 \\
\hline $\begin{array}{l}\mathrm{S}_{\mathrm{po}_{2}} \text { on discharge } \% \\
\text { Spirometry }\end{array}$ & $98.7 \pm 1.0$ & $98.6 \pm 1.2$ & $98.7 \pm 1.0$ & $98.5 \pm 1.0$ & 0.73 \\
\hline FVC \% pred & $93.59 \pm 12.25$ & $94.06 \pm 10.48$ & $94.12 \pm 12.31$ & $91.12 \pm 14.30$ & 0.632 \\
\hline FVC $<80 \%$ pred & $10(9.09)$ & $3(12.50)$ & $5(7.46)$ & $2(10.53)$ & 0.644 \\
\hline $\mathrm{FEV}_{1} \%$ pred & $92.70 \pm 11.57$ & $94.26 \pm 11.00$ & $92.59 \pm 11.87$ & $91.12 \pm 11.58$ & 0.676 \\
\hline $\mathrm{FEV}_{1}<80 \%$ pred & $15(13.64)$ & $4(16.67)$ & 9 (13.43) & 2 (10.53) & 0.857 \\
\hline $\mathrm{FEV}_{1} / \mathrm{FVC} \%$ & $80.70 \pm 5.81$ & $81.84 \pm 5.48$ & $80.39 \pm 6.12$ & $80.19 \pm 5.15$ & 0.509 \\
\hline $\mathrm{FEV}_{1} / \mathrm{FVC}<70 \%$ & $5(4.55)$ & $0(0)$ & $5(7.46)$ & $0(0)$ & 0.349 \\
\hline MMEF \% pred & $97.40 \pm 26.23$ & $99.77 \pm 28.17$ & $96.59 \pm 26.51$ & $96.14 \pm 23.82$ & 0.879 \\
\hline MMEF <65\% pred & $7(6.42)$ & $1(4.17)$ & 6 (9.09) & $0(0)$ & 0.551 \\
\hline $\mathrm{FEF}_{50 \%} \%$ pred & $94.74 \pm 26.11$ & $97.47 \pm 25.48$ & $94.09 \pm 26.80$ & $93.53 \pm 25.56$ & 0.845 \\
\hline $\mathrm{FEF}_{50 \%}<65 \%$ pred $^{\text {I }}$ & $12(11.01)$ & 2 (8.33) & 8 (12.12) & $2(10.53)$ & 1 \\
\hline $\mathrm{FEF}_{75 \%} \%$ pred & $96.10 \pm 32.56$ & $102.23 \pm 40.20$ & $95.02 \pm 30.89$ & $92.08 \pm 27.92$ & 0.549 \\
\hline $\mathrm{FEF}_{75 \%}<65 \%$ pred & $12(11.01)$ & $3(12.50)$ & $4(6.06)$ & $5(26.32)^{\#}$ & 0.035 \\
\hline \multicolumn{6}{|l|}{ Diffusion capacity } \\
\hline$D_{\text {Lco }} \%$ pred & $78.18 \pm 14.29$ & $84.70 \pm 13.88$ & $79.76 \pm 11.99$ & $64.79 \pm 14.35^{* *}, \# \#$ & $<0.001$ \\
\hline$D_{\text {LCO }}<80 \%$ pred & $51(47.22)$ & $7(30.43)$ & $28(42.42)$ & $16(84.21)^{* *, \# \#}$ & 0.001 \\
\hline$D_{\mathrm{LCO}} / V_{\mathrm{A}} \%$ pred & $92.09 \pm 16.68$ & $99.35 \pm 18.25$ & $92.30 \pm 15.70$ & $82.58 \pm 13.91 * *$ & 0.004 \\
\hline$D_{\mathrm{Lco}} / V_{\mathrm{A}}<80 \%$ pred & 29 (26.85) & $3(13.04)$ & $18(27.27)$ & $8(42.11)$ & 0.09 \\
\hline \multicolumn{6}{|l|}{ Lung volume } \\
\hline TLC \% pred & $86.32 \pm 11.32$ & $87.13 \pm 10.43$ & $88.11 \pm 10.72$ & $79.16 \pm 12.13^{*, \# \#}$ & 0.008 \\
\hline TLC $<80 \%$ pred & $27(25.00)$ & $4(17.39)$ & $14(21.21)$ & $9(47.37)^{*, \#}$ & 0.049 \\
\hline RV \% pred & $86.83 \pm 19.37$ & $87.17 \pm 16.88$ & $89.79 \pm 19.21$ & $76.16 \pm 19.96^{\# \#}$ & 0.024 \\
\hline $\mathrm{RV}<65 \%$ pred & $10(9.26)$ & $2(8.70)$ & $3(4.55)$ & $5(26.32)^{\#}$ & 0.021 \\
\hline RV/TLC $\%$ pred & $96.99 \pm 16.72$ & $98.00 \pm 14.93$ & $98.53 \pm 17.55$ & $90.42 \pm 14.86$ & 0.168 \\
\hline
\end{tabular}

Data are presented as $\mathrm{n}$, mean \pm SD or $\mathrm{n}(\%)$, unless otherwise stated. Comparisons between continuous variables were performed with one-way ANOVA. Chi-squared test and Fisher's exact test were applied to categorical variables as appropriate. BMI: body mass index; $S_{\mathrm{pO}_{2}}$ : oxygen saturation measured by pulse oximetry; FVC: forced vital capacity; $\mathrm{FEV}_{1}$ : forced expiratory volume in $1 \mathrm{~s}$; MMEF: maximal mid-expiratory flow; $\mathrm{FEF}_{50 \%}$ : forced expiratory flow at $50 \%$ of $\mathrm{FVC}_{;} \mathrm{FEF}_{75 \%}$ : forced expiratory flow at $75 \%$ of $\mathrm{FVC} ; D_{\mathrm{LCO}}$ : diffusing capacity of the lung for carbon monoxide; $D_{\mathrm{LCO}} / V_{\mathrm{A}}: D_{\mathrm{LCO}}$ corrected for alveolar volume; TLC: total lung capacity; RV: residual volume. ${ }^{*}$ : $p<0.05$ versus mild illness; ${ }^{* *}$ : $p<0.01$ versus mild illness; ${ }^{\#}: p<0.05$ versus pneumonia; ${ }^{\# \#}$ : $p<0.01$ versus pneumonia; " : at least two of these $<65 \%$ pred defined patients with small airway function anomalies.

This trend of the gradual decrease in level of $D_{\mathrm{LCO}}$ among patients was identical with the varying degree of severity. For about half (25 out of 51 ) of the $D_{\mathrm{LCO}}$-impaired patients, the $D_{\mathrm{LCO}}$ corrected for alveolar volume $\left(D_{\mathrm{LCO}} / V_{\mathrm{A}}\right)$ was still within the normal range, which might indicate that the $D_{\mathrm{LCO}}$ decrease was more than the $D_{\mathrm{LCO}} / V_{\mathrm{A}}$ decrease, in recovered subjects. The value of TLC $\%$ pred in severe pneumonia cases was much less than that of pneumonia or mild illness cases, suggesting higher impairment of lung volume in severe cases. There was no significant difference among the discharged survivors with different severity in regard to other ventilatory defects (e.g. $\mathrm{FEV}_{1}, \mathrm{FVC}, \mathrm{FEV}_{1} / \mathrm{FVC}$ ). 
Recent studies reveal that the lung is the organ most affected by COVID-19 [2, 3], with pathologies that include diffuse alveolar epithelium destruction, capillary damage/bleeding, hyaline membrane formation, alveolar septal fibrous proliferation, and pulmonary consolidation. Previous studies have demonstrated that recovered patients with coronavirus pneumonia can be left with damaged lungs. Impaired lung function was common and could last for months or even years. In the follow-up studies lasting 0.5-2 years in rehabilitating SARS patients [5-7], impaired $D_{\text {LCO }}$ was the most common abnormality, ranging from $15.5 \%$ to $43.6 \%$, followed by defective TLC, ranging from $5.2 \%$ to $10.9 \%$. PARK et al. [8] showed that $37 \%$ of MERS survivors still presented with an impairment of $D_{\mathrm{LCO}}$, but normal TLC, at 12 months. Our study seems to be more consistent with the findings in SARS. Interestingly, in our study, the greater decline in $D_{\mathrm{LCO}}$ versus $D_{\mathrm{LCO}} / V_{\mathrm{A}}$ suggests that the diffusion membrane may be more causative of the pulmonary dysfunction compared to lowered lung volume. The low proportion and severity of small airway dysfunction in our cohort also suggests that COVID-19 is more likely associated with diffuse lung epithelial damage and small airway congestion. when evaluating lung fibrotic changes in SARS, the dynamic $D_{\text {LCO }}$ scores were found to be more sensitive than high-resolution CT [11]. Whether survivors of COVID-19 with impairment of $D_{\mathrm{LCO}}$ or residual abnormalities on chest CT will develop pulmonary fibrosis requires further investigation.

There are limitations in our study. First, the lack of baseline pulmonary function test results prior to the illness make it difficult to make a comparison with the results after the illness. There were only a minority of patients with chronic respiratory disease, so it should be acceptable to speculate that the basic lung function in the majority of patients would be normal. The interpretation regarding the impact of COVID-19 on lung function remains valid. Secondly, the association between CT images and the lung function parameters was not analysed in our study. Finally, this cross-sectional analysis only provides a short follow-up, and the long-term dynamic variation of lung function after hospital discharge still requires further investigation.

In conclusion, our study reveals that, in discharged survivors with COVID-19, impairment of diffusion capacity is the most common abnormality of lung function, followed by restrictive ventilatory defects, which are both associated with the severity of the disease. Pulmonary function tests (not only spirometry but also diffusion capacity) should be considered in routine clinical follow-up for certain recovered survivors, especially in severe cases. Subsequent pulmonary rehabilitation might be considered as an optional strategy. Long-term studies are needed to address whether these deficits are persistent.

Xiaoneng Mo ${ }^{1,4}$, Wenhua Jian ${ }^{2,4}$, Zhuquan $\mathrm{Su}^{2,4}$, Mu Chen ${ }^{1}$, Hui Peng ${ }^{1}$, Ping Peng ${ }^{1}$, Chunliang Lei ${ }^{3}$, Ruchong Chen ${ }^{2,5}$, Nanshan Zhong ${ }^{2,5}$ and Shiyue $\mathrm{Li}^{2,5}$

${ }^{1}$ Dept of Respiratory Medicine, Guangzhou Eighth People's Hospital, Guangzhou, China. ${ }^{2}$ China State Key Laboratory of Respiratory Disease, National Clinical Research Center for Respiratory Disease, Guangzhou Institute of Respiratory Health, First Affiliated Hospital of Guangzhou Medical University, Guangzhou, China. ${ }^{3}$ Dept of Hepatology, Guangzhou Eighth People's Hospital, Guangzhou, China. ${ }^{4}$ Xiaoneng Mo, Wenhua Jian and Zhuquan Su are joint first authors. ${ }^{5}$ Ruchong Chen, Nanshan Zhong and Shiyue Li are joint corresponding authors.

Correspondence: Shiyue Li, China State Key Laboratory of Respiratory Disease, National Clinical Research Center for Respiratory Disease, First Affiliated Hospital of Guangzhou Medical University, Guangzhou, 510120, China. E-mail: lishiyue@188.com

Received: 15 April 2020 | Accepted after revision: 28 April 2020

Published in volume 55, issue 6 of the European Respiratory Journal on 18 June 2020; republished 16 October 2020 with amendments to the authors' affiliation details.

Acknowledgements: We thank the hospital staff for their efforts in collecting the data. We also thank Weijie Guan, Yi Gao, Zhe Zhang, Jinping Zheng and Guangqiao Zeng (First Affiliated Hospital of Guangzhou Medical University) for critical opinion. None of these individuals received compensation for their contributions.

Author contributions: S. Li, R. Chen and N. Zhong contributed equally as senior authors. S. Li, R. Chen and N. Zhong had full access to all of the data in the study and take responsibility for the integrity of the data and the accuracy of the data analysis. Concept and design: X. Mo, R. Chen, S. Li. Acquisition, analysis, or interpretation of data: X. Mo, W. Jian, Z. Su, M. Chen, H. Peng, P. Peng, C. Lei, R. Chen, S. Li. Drafting of the manuscript: X. Mo, W. Jian, Z. Su, R. Chen. Critical revision of the manuscript for important intellectual content: C. Lei, N. Zhong.

Conflict of interest: None declared.

Support statement: This study was supported by the National Key R \& D Program of China (2018YFC1311900) and the National Science Foundation of China (No. 81770017). The funding organisations had no role in the design and conduct of the study.

\section{References}

1 World Health Organization. Coronavirus Disease 2019 (COVID-19) Situation Report - 93. www.who.int/docs/defaultsource/coronaviruse/situation-reports/20200422-sitrep-93-covid-19.pdf?sfvrsn=35cf80d7_4 Date last updated: 22 April 2020. 
2 Zhou F, Yu T, Du R, et al. Clinical course and risk factors for mortality of adult inpatients with COVID-19 in Wuhan, China: a retrospective cohort study. Lancet 2020; 395: 1054-1062.

3 Xu Z, Shi L, Wang Y, et al. Pathological findings of COVID-19 associated with acute respiratory distress syndrome. Lancet Respir Med 2020; 8: 420-422.

4 Wang Y, Dong C, Hu Y, et al. Temporal changes of CT findings in 90 patients with COVID-19 pneumonia: a longitudinal study. Radiology 2020; in press [https://doi.org/10.1148/radiol.2020200843].

5 Hui DS, Joynt GM, Wong KT, et al. Impact of severe acute respiratory syndrome (SARS) on pulmonary function, functional capacity and quality of life in a cohort of survivors. Thorax 2005; 60: 401-409.

6 Hui DS, Wong KT, Ko FW, et al. The 1-year impact of severe acute respiratory syndrome on pulmonary function, exercise capacity, and quality of life in a cohort of survivors. Chest 2005; 128: 2247-2261.

7 Ngai JC, Ko FW, Ng SS, et al. The long-term impact of severe acute respiratory syndrome on pulmonary function, exercise capacity and health status. Respirology 2010; 15: 543-550.

8 Park WB, Jun KI, Kim G, et al. Correlation between pneumonia severity and pulmonary complications in Middle East respiratory syndrome. J Korean Med Sci 2018; 33: e169.

9 World Health Organization. Clinical management of severe acute respiratory infection when COVID-19 is suspected. www.who.int/publications-detail/clinical-management-of-severe-acute-respiratory-infection-when-novelcoronavirus-(ncov)-infection-is-suspected Date last updated: 13 March 2020

10 National Health Commission \& State Administration of Traditional Chinese Medicine. Diagnosis and Treatment Protocol for Novel Coronavirus Pneumonia (Trial Version 7). www.who.int/docs/default-source/wpro--documents/countries/china/covid-19-briefing-nhc/1-clinical-protocols-for-the-diagnosis-and-treatment-of-covid-19v7.pdf?sfvrsn=c6cbfba4_2 Date last updated: 3 March 2020.

11 Xie L, Liu Y, Xiao Y, et al. Follow-up study on pulmonary function and lung radiographic changes in rehabilitating severe acute respiratory syndrome patients after discharge. Chest 2005; 127: 2119-2124. 